\title{
RNA unwinding from reweighted pulling simulations
}

\author{
Francesco Colizzi* and Giovanni Bussi* \\ SISSA - Scuola Internazionale Superiore di Studi Avanzati, \\ via Bonomea 265, 34136 Trieste, Italy \\ E-mail:colizzi@sissa.it; bussi@sissa.it
}

\begin{abstract}
The forming and melting of complementary base pairs in RNA duplexes are conformational transitions required to accomplish a plethora of biological functions. Yet the dynamic steps of these transitions have not been quantitatively characterized at the molecular level. In this work, the base opening process was first enforced by atomistic pulling simulations and then analyzed with a novel reweighting scheme which allowed the freeenergy profile along any suitable reaction coordinate, e.g. solvation, to be reconstructed. The systematic application of such approach to different base-pair combinations provides a molecular motion picture of helix opening which is validated by comparison with an extensive set of experimental observations and links them to the enzyme-dependent unwinding mechanism. The RNA intrinsic dynamics disclosed in this work could rationalize the directionality observed in RNA-processing molecular machineries.
\end{abstract}

\section{Introduction}

The ability of ribonucleic acid (RNA) to adopt peculiar three-dimensional structures that mediate a variety of biological functions makes it the most versatile regulatory factor in the cell.$^{1}$ Virtually involved in all cellular processing of the genetic information, the RNA is able to achieve such a functional diversity by adaptively acquiring very distinct conformations in response to specific conditions of the cellular environment. ${ }^{[2}$ Among the

${ }^{*}$ To whom correspondence should be addressed structural rearrangements engaged by RNA, the opening of complementary base pairs is an ubiquitous process required to accomplish a wide range of metabolic activities such as transcription, premRNA splicing, ribosome biogenesis or translation initiation. ${ }^{[3}$ In the cell this is usually catalyzed by enzymes called RNA helicases which have been shaped by the evolution to unwind doublestranded (ds) RNA according to its intrinsic dynamic properties. 45

From a molecular standpoint, the opening and forming of individual base pairs are fundamental, yet poorly understood, events which provide the structural framework to large-scale RNA conformational transitions and folding. ${ }^{26-12]}$ In this respect and related to the work presented herein, insightful investigations have been reported only for short deoxyribonucleic acids (DNAs) in the Bform helical geometry. 13 Using transition-path sampling, Hagan et al ${ }^{14}$ have fully characterized the energetics of (un)pairing for a $5^{\prime}$-end cytosine. However, the mechanism underlying the complete opening of the duplex has not been systematically faced nor analyzed. Moreover, differences in topology and thermodynamic parameters between B-form DNA and A-form RNA suggest that the mechanism of duplex separation might obey different rules.

Recently, combining thermodynamic information with the relative population of unpaired terminal nucleotides (dangling ends) observed in large ribosomal RNA (rRNA) crystal structures, Mohan et al ${ }^{16}$ have proposed that stacking and pairing reactions are not simultaneous, and that $3^{\prime}$ single-strand stack leads the base pairing of the 5'-strand. Nevertheless, collecting an unbiased 
data set of dangling-end population is not trivial and, when viewed in the context of the full ribosomal assemblies, the single stranded (ss) regions are seen to interact extensively with other RNA elements. ${ }^{16}$ On top of that, since the formation and opening of base pairs is a dynamic process, both the ensemble-averaged thermodynamic properties and the detailed but static X-ray picture have to be complemented with other methods able to directly and quantitatively capture the dynamics of the investigated event. Likely, this gap will be efficiently bridged by ad hoc designed spectroscopic approaches. ${ }^{2|9| 12 \mid 17}$ For instance, femtosecond timeresolved fluorescence spectroscopy is emerging as a powerful technique for the quantitative analysis of base-stacking pattern and base motion, 18 although its applications to probe RNA dynamics are still in their infancy and the method presents several limitations. 11 As a matter of fact, the integration of spectroscopic approaches with other powerful techniques is presently needed to gain molecular details on the RNA intrinsic dynamics. Among the possible methodological choices, atomistic simulations 19 allow any base sequence to be characterized and all the microscopic parameters to be controlled. Additionally, when combined with state-of-the-art free-energy methods, they can provide an unparalleled perspective on the mechanism and dynamics of the biomolecular process of interest. As far as the reconstruction of free-energy profiles is concerned, the capability of estimating those profiles along any suitable reaction coordinate, without any further computational cost, would offer researchers a powerful and versatile tool enabling both the disclosure of intermediate states and the multifaceted analysis of complex conformational transitions.

With this spirit, here we report an in silico study elucidating the mechanism for strand separation in the RNA double helix. In particular, we used atomistic steered molecular dynamics (MD) simulations ${ }^{20}$ to enforce the unbinding of nucleobases into the surrounding explicit water. To allow a systematic analysis of different base sequences we devised a novel Jarzynski-equation-based reweighting scheme which allowed the free-energy landscape to be reconstructed as a function of different reaction coordinates and the unbinding energies to be straightforwardly estimated. The computed free-energy differences are consistent with experimental observations and suggest that the strand separation mechanism occurs by a stepwise process in which the probability of unbinding of the base at the $5^{\prime}$ terminus is significantly higher than that at the $3^{\prime}$ terminus. The biological implications of these findings are discussed and related to the unwinding mechanism catalyzed by RNA processing machineries. Given the general nature of our approach, the introduced methodology can be directly applied to analyze a broad range of molecular unbinding processes.

\section{Methods}

Throughout the manuscript the following nomenclature will be consistently used to define each elementary step involving one single base and occurring during the opening of a closed base pair: unpairing is used to define the process undergone by a single base for which both Watson-Crick hydrogen bonds and stacking interactions with adjacent bases are broken; unstacking is the process breaking the stacking interactions between a dangling terminal nucleotide and its adjacent bases. The opening of a base pair is thus composed by an initial unpairing followed by an unstacking (1). In the manuscript we also use the term unbinding referring to both unpairing and unstacking processes by no means of specificity. Finally, the strand with the $5^{\prime}$ terminal (or $3^{\prime}$ terminal) nucleobase being pulled is referred to as the $5^{\prime}$-strand (or $3^{\prime}$-strand).

\section{System set-up}

We simulated the unpairing and unstacking of nucleobases at both $3^{\prime}$ - and $5^{\prime}$-termini in dsRNAs of sequence 5'-CCGGGC-3', and 5'-GGCCCG-3' $^{\prime}$ '-GGCCG-5' (2). Two sets of data can be obtained from each dsRNA thus resulting in four systems with different combinations of Watson-Crick base pairing and stacking (2A). Both terminal and nonterminal base pairs (i.e. a base pair at the ss-ds RNA junction) were investigated. Non-terminal base pairs showed the same trend in relative stability observed for terminal ones, and are reported in the Supporting Information (SI). The A-form 


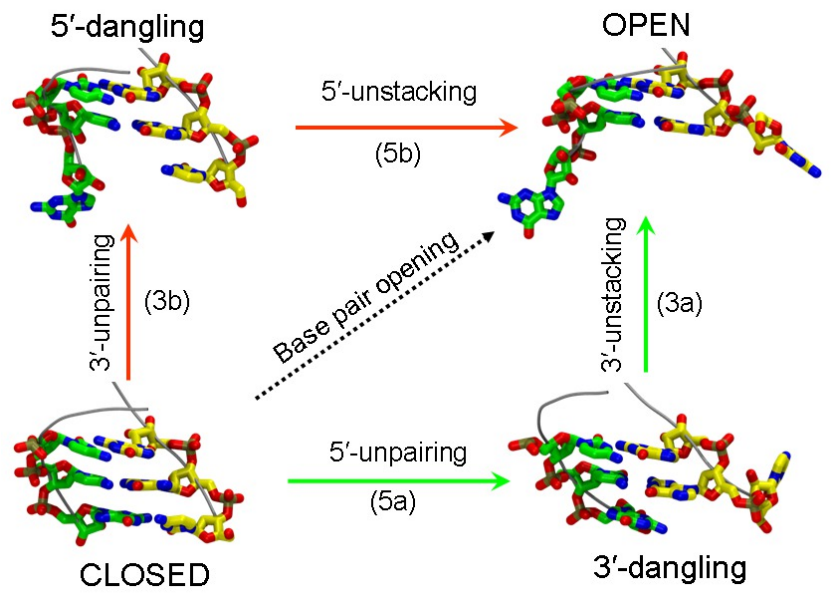

Figure 1: Elementary steps involved in the opening of a base pair. The thermodynamics cycle was used to characterize different base pair combinations.

dsRNA was built using ASSEMBLE ${ }^{21}$ and then solvated with $\sim 3600$ water molecules, $20 \mathrm{Na}^{+}$and $10 \mathrm{Cl}^{-}$ions, resulting in an excess salt concentration of about $0.15 \mathrm{M}$. The mobility of added ions was fairly diffusive during the simulations. After minimization and thermalization, each system (or intermediate) was then evolved for $30 \mathrm{~ns}$ in the isothermal-isobaric ensemble $(300 \mathrm{~K}, 1 \mathrm{Atm})^{22223}$ using the Amber99 force field ${ }^{24}$ and TIP3P water. ${ }^{[25}$ Preliminary calculations carried out using the recent refinement of the Amber99 force field $(\text { parmbsc } 0)^{26}$ have shown quantitatively similar results in the reconstructed free-energy profiles. This is probably due to the poor involvement of the refined $\alpha$ and $\gamma$ dihedrals during the unbinding trajectories. Long-range electrostatic interactions were calculated with the particle mesh Ewald method. ${ }^{[27}$ Plain MD and biased steered MD trajectories were generated with GROMACS 4.0.728 combined with PLUMED 1.2. ${ }^{29}$

\section{Pulling simulations}

The starting configurations for the pulling simulations were randomly sampled from the corresponding $30 \mathrm{ns-long}$ runs. The distance between the center of mass of two stacked bases (2B) was used as pulled collective variable $(\mathrm{CV})$, and thus harmonically restrained to a constant-velocity moving point, starting at a position equal to the equilibrium average of the $\mathrm{CV}$ and pulling it by

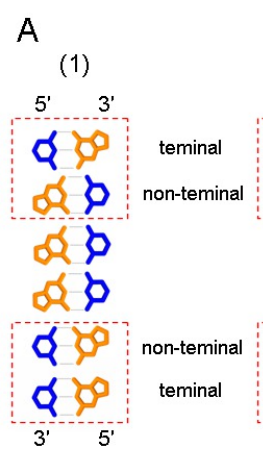

(3)

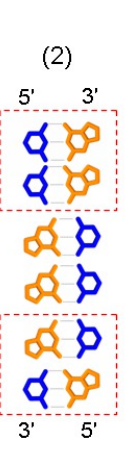

(4)

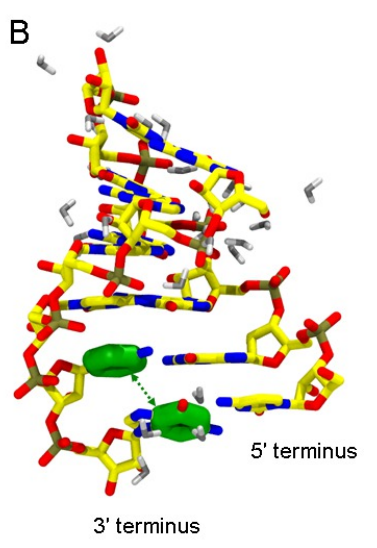

Figure 2: RNA double helix. A) Schematic view of the combinations (red dotted boxes) of guanine (orange) and cytosine (blue) base-pairing and -stacking investigated. B) Structural representation of the RNA duplex in water; the distance between the center of mass (green arrow) of the sixmembered ring atoms (thick green sticks) of two stacked bases was used as collective variable for the pulling simulations.

$0.75 \mathrm{~nm}$ in $1.5 \mathrm{~ns}$. This resulted in a biasing potential equal to $V_{\text {bias }}(q, t)=\frac{k}{2}\left[s(q(t))-\left(s_{0}+v t\right)\right]^{2}$ where $k=1200 \mathrm{kcal} / \mathrm{mol} / \mathrm{nm}^{2}$ is the spring constant of the restraint, $q$ are the microscopic coordinates, $s(q)$ is the $\mathrm{CV}$ value for those coordinates, $s_{0}$ is the initial position of the restraint, $v$ is the pulling velocity and $t$ is the time.

The mechanical work done during the process was obtained by integrating the force exerted on the system along the biased reaction coordinate. After collecting about 400 realizations for each nucleobase-unbinding process, the Jarzynski nonequilibrium work theorem ${ }^{30}$ was exploited to discount the dissipated work and to reconstruct the free-energy profile as a function of the restraint distance $\left(s_{0}+v t\right)$. Although employing Jarzynski's equality in principle allows unbiased free-energy differences to be estimated, its direct application is limited by the number of collectable realizations as well as by the complexity of the system. ${ }^{[31}$ A typical free-energy profile is shown in $3 \mathrm{~A}$ as a function of the restraint distance. The blue plot shows how, after a steep rise, a series of alternating shoulders and local plateaus gradually brought the system to higher free-energy states. Moreover, between distances ranging from 1 to $1.2 \mathrm{~nm}$ (for the exemplified system), the profile was strongly dominated 

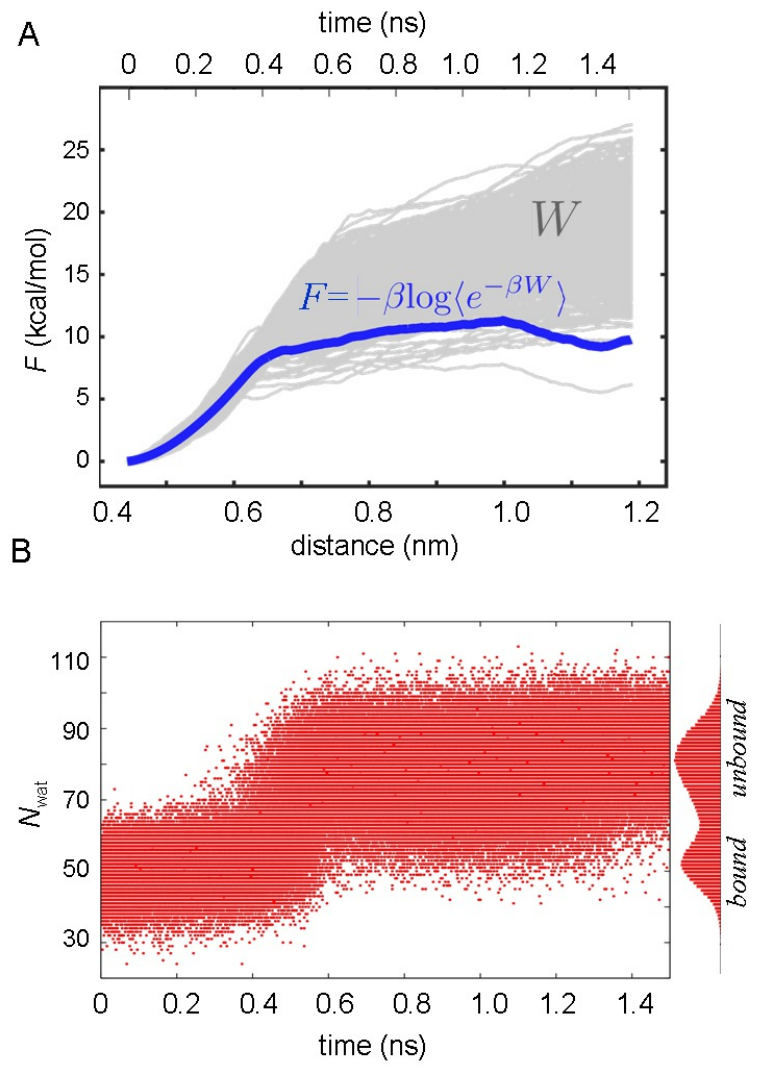

Figure 3: Typical nucleobase unbinding process, obtained by pulling along the distance between the center of mass of two stacked bases. A) Mechanical work (W) performed (gray plot) and its exponential average (blue plot) as in Jarzynski equality, plotted as a function of the restraint position. The distance is practical for biasing the system but hardly allowed defining the bound and unbound states. B) Number of water molecules coordinating the unbinding nucleobase $\left(N_{\text {wat }}\right)$ as a function of time (main panel), and its probability distribution (right panel). The coordination with water is an useful metrics for identifying the bound and unbound states.

by an outlier low-work realization and the difference in reconstructing the free-energy profile with or without the outlier was more than $3 \mathrm{kcal} / \mathrm{mol}$ (see SI).

Within this framework, there was no clean way to automatically detect when the nucleobase had reached the unbound configuration, and it was difficult to avoid systematic errors in the comparison of many profiles with small differences as we were interested in. To tackle this problem, we decided to analyze our simulations in terms of CVs differ- ent from the one used for the pulling. This a posteriori analysis could be done quickly, as a postprocessing, and allowed us to choose optimal CVs capable of describing in an user-independent manner all the unbinding events.

\section{Reweighting scheme}

To project the free-energy landscape on putative CVs we devised a proper reweighting scheme. Whereas suitable schemes have been proposed to reweight other types of nonequilibrium simulations (e.g. Ref. ${ }^{32}$ ), a reweighting algorithm for steered MD has not been reported. For a different purpose, Hummer and Szabo developed a method which enables the reconstruction of the free energy as a function of the pulled coordinate. ${ }^{33 / 34}$ Here, we generalize this scheme so as to compute the free energy as a function of any a posteriori chosen variable.

Two different sorts of bias affect the steered MD trajectories and needed to be removed: (a) the nonequilibrium nature of the pulling and (b) the presence of artificial harmonic restraints on the pulled CV. The nonequilibrium bias is removed by noticing that the equilibrium probability $P_{\text {eq }}(q, t)$, for a restraint statically kept in its position at time $t$, can be obtained from the non-equilibrium one $P_{\text {neq }}^{(i)}(q, t)$ as observed in the $i$-th trajectory exploiting a relation first reported by Crooks: $\underline{.356}$

$$
P_{\mathrm{eq}}(q, t)=\sum_{i} e^{-\beta\left[W_{i}(t)-F(t)\right]} P_{\text {neq }}^{(i)}(q, t)
$$

where $W_{i}(t)$ is the work done on the $i$-th trajectory up to time $t$ and $\beta=1 / k_{B} T$ is the inverse thermal energy. Here the free energy $F(t)$ represents the normalization factor corresponding to the instantaneous position of the moving restraint at time $t$. The bias of the harmonic restraint can then be removed by applying the weighted-histogram analysis method. $\frac{37}{}$ Whereas weighted histograms are traditionally used to combine independent simulations performed with different static biasing potentials, here we used it to combine snapshots obtained at different stages of the pulling, thus writ- 
ing the unbiased equilibrium probability as

$$
P_{\mathrm{u}}(q) \propto \frac{\int_{0}^{\tau} d t P_{\mathrm{eq}}(q, t)}{\int_{0}^{\tau} d t e^{-\beta[V(q, t)-F(t)]}},
$$

where $\tau$ is the length of each pulling simulation. Finally, the free energy as a function of an arbitrary, a posteriori chosen CV $\bar{s}$ is defined as $F(\bar{s})=$ $-k_{B} T \log \int d q P_{u}(q) \delta(\bar{s}-\bar{s}(q))$. The scheme described so far closely resembles the one used by Hummer and Szabo. ${ }^{33}$ However, it is conceptually different, as here the free energy can be reconstructed also with respect to a variable different from the pulled one. Thus, it potentially enables the disclosure and characterization of otherwise hidden features of the investigated process. To further simplify the data manipulation and to avoid building multidimensional histograms, with a further dependence on technical choices such as binning size, we recast our approach assigning a weight to each of the sampled configurations, in the same spirit as in Ref. 38 . After simple manipulation, the weight can be shown to be equal to

$$
w_{i}(t) \propto \frac{e^{-\beta\left[W_{i}(t)-F(t)\right]}}{\int_{0}^{\tau} d t^{\prime} e^{-\beta\left[V\left(q_{i}(t), t^{\prime}\right)-F\left(t^{\prime}\right)\right]}} .
$$

The normalization factor for each time, $F(t)$, is then computed iteratively up to convergence as $e^{-\beta F(t)}=\sum_{i} \int_{0}^{\tau} d t^{\prime} w_{i}\left(t^{\prime}\right) e^{-\beta V\left(q_{i}\left(t^{\prime}\right), t\right)}$. Usually a few tens of iterations are enough to converge.

In summary, in our reweighting scheme we first compute the weight of each of the configurations saved along the MD simulations from 3 , then estimate free energies as a function of any, a posteriori chosen $\mathrm{CV}$ as

$$
F(\bar{s})=-k_{B} T \log \sum_{i} w_{i}(t) \delta\left(\bar{s}-\bar{s}\left(q_{i}(t)\right)\right.
$$

\section{Results}

Using the reweighting scheme outlined in the previous Section we were able to investigate several order parameters. Since solvent interactions are known to affect the conformational state of nucleic acids,,$\frac{39}{}$ we considered the solvation of an unbinding base as an effective metrics for the progression of the underlying process. This choice al- lowed defining the unbinding in a manner which was totally independent from both the terminus and the specific base, and, in our explicit-solvent simulation, could be computed as the coordination among heavy atoms of the base and water oxygens (3B). In this metrics, the bound and unbound states could be clearly and unambiguously identified and corresponded to approximately harmonic basins. Sample free energies computed as a function of the number of water molecules coordinating the unpairing base are shown in 4 . The free-energy profile reconstructed along such a reaction coordinate is in no way biased by the absolute number of coordinated water molecules which is merely used to distinguish one configuration from the other and to properly collect the corresponding weights along the simulation as in 4. Then, to compute accurately the bound/unbound free-energy differences, we fit the free-energy profiles with the combination of two quadratic functions,, $40[41$

$$
e^{-\beta F(\bar{s})}=\sigma_{1}^{-1} e^{-\frac{\left(\bar{s}-\bar{s}_{1}\right)^{2}}{2 \sigma_{1}^{2}}-\beta F_{1}}+\sigma_{2}^{-1} e^{-\frac{\left(\bar{s}-\bar{s}_{2}\right)^{2}}{2 \sigma_{2}^{2}}-\beta F_{2}}
$$

where $F_{1}$ and $F_{2}$ are the free energies of bound and unbound states. Both, when the two states were clearly resolvable (e.g. 4, left panels), and when the corresponding $\mathrm{CV}$ population was more overlapped, (e.g. 4 right panels) the fitting procedure resulted robust and poorly sensitive to outlier work realizations, thus enhancing convergence of the results (e.g. the difference in performing the fit with or without the outlier low-work realization in 3A was less than $0.3 \mathrm{kcal} / \mathrm{mol}$, see SI). Furthermore, this approach showed very stable outcomes with respect to the choice of the details in the definition of the solvation order parameter, and allowed comparing systematically several similar situations without incurring of large statistical errors or, worst, human biases in the interpretation of the results.

Having an optimized statistical-mechanics tool able to provide free-energy differences in a flexible and automatic manner, we pursued a systematic step-by-step approach to investigate the feasibility of different opening paths for the four possible combinations of G-C base stacking and WatsonCrick pairing. The general procedure, as outlined in 1. relied on two subsequent steps: first, the 


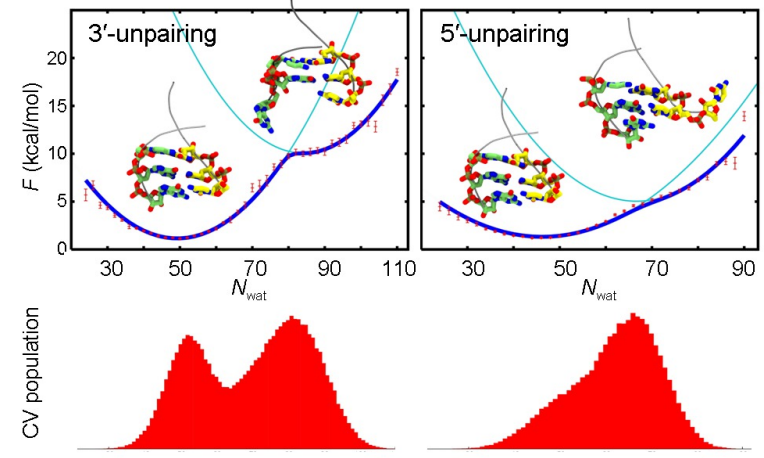

Figure 4: Reconstruction of the free-energy profile as a function of the number of water molecules $\left(N_{\text {wat }}\right)$ surrounding the unbinding base. In the left and right panels are shown typical free energy profiles (red dots with error bar) for the unpairing of a $3{ }^{\prime}$-strand guanine and $5^{\prime}$-strand cytosine, respectively. The quadratic potentials obtained from the double-well fitting are shown in light blue color, whereas their combination [5] is in blue. Underneath each panel, the unnormalized population of the $\mathrm{CV}$ is also shown.

Watson-Crick base pair was partially opened by the unpairing of the base on either the $5^{\prime}$ or $3^{\prime}$ terminus; second, the resulting dangling intermediate, on the $3^{\prime}$ or $5^{\prime}$ terminus respectively, was unstacked and the base pair opening completed.

Table 1: Context-dependent base-unbinding free energy $(\mathrm{kcal} / \mathrm{mol})$ corresponding to the elementary steps shown in 1 .

\begin{tabular}{|c|c|c|c|c|}
\hline Construct & \multicolumn{4}{|c|}{ Opening steps } \\
\hline$n$ & $3 b$ & $5 b$ & $5 a$ & $3 a$ \\
\hline (1) $)^{5^{\prime}-\mathrm{CG} . .}$ & 7.6 & 0.9 & 2.6 & 5.6 \\
\hline 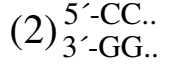 & 8.9 & 0.3 & 3.9 & 4.5 \\
\hline (3) $)^{5^{\prime}-\mathrm{GG} . .}$ & 7.0 & 2.2 & 4.9 & 3.9 \\
\hline 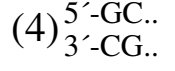 & 7.4 & 2.0 & 5.7 & 2.8 \\
\hline
\end{tabular}

The relative stability of putative intermediates involved in the opening of a base pair was estimated from the individual base-unbinding free energies (1) and 1). For all the considered combinations, the difference in basepair-opening free energy computed biasing the system along path (a) and (b) in 1 was lower than $1 \mathrm{kcal} / \mathrm{mol}$. For sake of clarity, it should be reminded that a finite number of unidirectional pulling simulations performed within a Jarzynski-like scheme are known to provide overestimates of absolute free-energy differences. 31 However, highly accurate estimates of unbinding constants were not needed to characterize the strand separation mechanism and the freeenergy differences we estimated were exploited as a quantitative tool to assess the relative stability of different configurations.

\section{Comparison with experiments}

Below we discuss the results of the first and second unbinding steps (1), and compare them with crystal structures conformer distributions, ${ }^{16 / 42}$ relative population of stacked/unstacked bases detected by femtosecond time-resolved fluorescence spectroscopy, 43 and thermodynamic data based on dsRNA melting experiments. ${ }^{14445}$ The consistency with experimental observations and the capability of our simulations to complement those results are highlighted.

The more general outcome arising from the comparison of free-energy differences is that the paired base on the $5^{\prime}$ terminus always interacted more weakly than the complementary base on the $3^{\prime}$ terminus (steps 5a, 3b in 1 and 1). This could be directly related to the A-form helical geometry of RNA in which the bases at the $5^{\prime}$ end of a ss-ds junction are less buried into the neighboring environment and expose a wider portion of their surface to water molecules, thus facilitating fraying events. The different stability of the nucleobase on the $5^{\prime}$ terminus can be reflected in the probability of observing a certain type of blunt closing base pair at ss-ds junctions. In this context, the stronger interaction was estimated for the $5^{\prime}$ guanine in ${ }^{5}$-GC... (construct 4 ) which was $\sim 1.7$ $\mathrm{kcal} / \mathrm{mol}$ weaker than the complementary cytosine on the 3'-terminus. Accordingly, the combination ${ }^{5}$-GC.. (construct 4 ) is the most abundant closing base-pair pattern observed at ss-ds junctions in large rRNA crystal structures. $\frac{16}{16}$ It can be further noticed that among the dangling ends (steps $5 b$ and $3 a$ in 1 and 1 ) the most stable ones are those on the $3^{\prime}$ terminus, consistently with ultrafast spectroscopy experiments which have detected a large subpopulation of stacked conform- 
ers for a $3^{\prime}$-dangling fluorescent purine probe, while only a relatively small one for a $5^{\prime}$-dangling purine probe. ${ }^{43}$ In particular, we found the most stable $3^{\prime}$-dangling end in construct $1\left(\begin{array}{c}5^{\prime}-C_{\mathrm{G}} . . \\ 3^{\prime}-\mathrm{GC} .\end{array}\right)$, which has also been counted as the most common dangling end pattern in rRNA crystal structures. 16 Further agreement can be found considering dsRNA optical melting experiments which have shown that single-nucleotides overhanging at 3 '-ends of an RNA helix increase the stability of the duplex in a sequence-dependent manner. Notably, such a stabilization has been interpreted as the capability of the $3^{\prime}$ dangling ends to stack over the hydrogen bonds of the closing base pair protecting them from water exchange. 46 Those 3'-dangling bases which are more likely stacked would thus provide a larger contribution to duplex stabilization. In this light, the trend that we observed in the unstacking energies of the four dan-

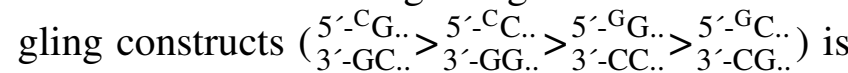
in agreement with duplex stabilization observed in dsRNA melting experiments. $\frac{144 \mid 45}{}$ It should be remarked that the duplex stabilization induced by 5 '-dangling ends might not reflect the stacking energy of the dangling end itself because of its small overlap with the hydrogen bonds of the closing base pair. Further discussion on the comparison of computed and experimental dangling-end stabilities can be found in the SI.

Summarizing, the unbinding of the base on the $5^{\prime}$-strand was, in all the considered cases, favored over the unbinding of the complementary $3^{\prime}$-strand base. Whereas the relative probability of $3^{\prime}$ - and $5^{\prime}$-unbinding event can be modulated by the sequence, the general trend remains unchanged.

From a structural standpoint, the unpairing could proceed through two qualitatively different paths: one in which the twisting and breaking of WatsonCrick hydrogen bonds occurred before the rupture of stacking interactions; the other, in which the unbinding followed the concerted rupture of both hydrogen bonds and stacking interactions with, in some cases, the unbinding base stacking over the dangling end of the opposite strand (5). Similar unbinding geometries have also been described in other studies. ${ }^{14 / 47}$ In our simulations, these intermediate states occurred with a context-dependent frequency. For instance, along the unbinding path- way the $5^{\prime}$-terminal guanine in $5^{\prime}$-GC.. (construct 4) had $\sim 15 \%$ of probability to stack upon the 3 -dangling cytosine of the opposite strand. Interestingly, this probability dropped to $\sim 3 \%$ in 5'-GG.. (construct 3). Such an inter-strand stacking pattern (5, panel $\left.t_{2}\right)$, exchanging with the conventional stacking of a paired base, could account for the delayed quenching of fluorescence detected by ultrafast fluorescence spectroscopy for a construct

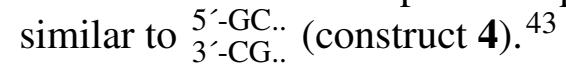

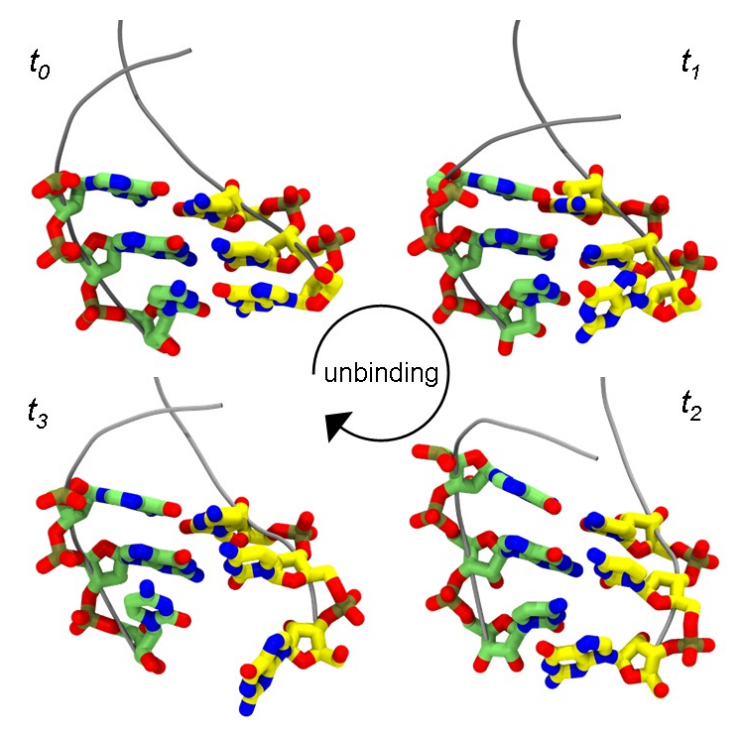

Figure 5: Snapshots sampled from the opening of a base pair. The unbinding base (here a 5 '-terminal guanine in construct 4) could transiently stack over the dangling end of the opposite strand $\left(t_{2}\right)$.

As final experimental evidences which corroborate our results, Xia and co-workers ${ }^{43}$ have reported that the dynamic behavior of a 3'-terminal purine is not affected by the presence of the opposite complementary base. Viceversa, the conformational dynamics of a $5^{\prime}$-terminal purine is drastically influenced by the presence of an opposite $3^{\prime}$-terminal pyrimidine which would be likely stacked and potentially able to shift the population of the complementary 5'-terminal base towards a paired and stacked ensemble. Consistently with our systematic study, these data depict the formation of a stable base pair as generally driven by the stacking of the $3^{\prime}$-terminal base, and then by the energy gained by the system from both the stacking of the 5'-terminal base and Watson-Crick hydrogen-bonds. 

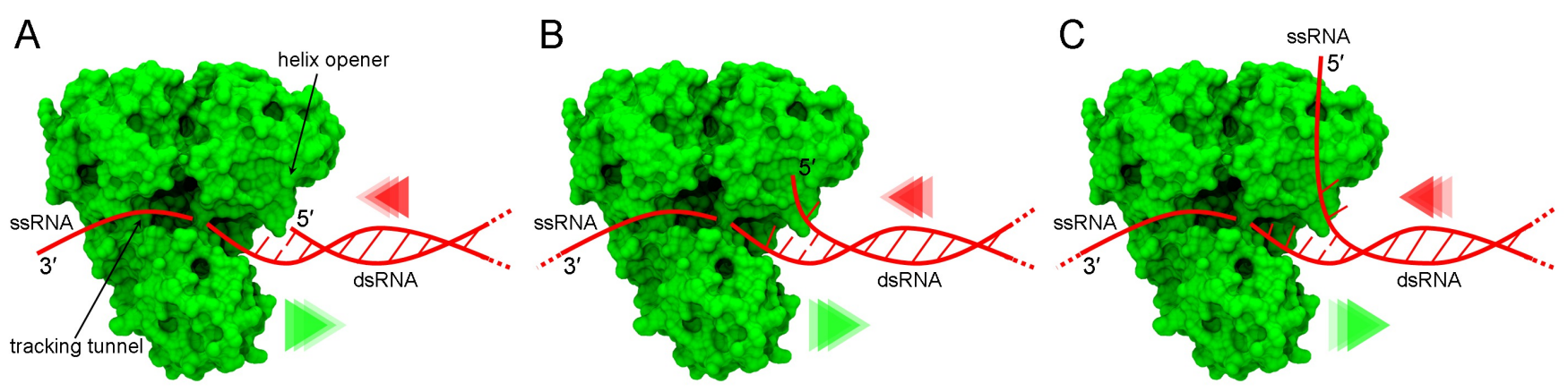

Figure 6: Model for RNA unwinding catalyzed by NS3 helicase. A) The single-stranded 3' terminus is loaded into the tracking tunnel. B) The $5^{\prime}$ terminus is mechanically displaced by the helix opener. C) The helicase proceeds with $3^{\prime}$ to $5^{\prime}$ directionality, displacing the $5^{\prime}$-strand.

\section{Biological implications}

The motion picture of duplex separation emerging from the outcome of our simulations complements and augments with dynamic details and energetic considerations the helix propagation model based on the analysis of static $3 \mathrm{D}$ structures. $\frac{16}{16}$ Our computations link experimental data from different fields creating a common reading frame among them. Taken together, these results suggest that RNA unwinding occurs by a stepwise process in which the probability of unbinding of the base on the $5^{\prime}$ strand is significantly higher than that on the 3' strand. What could be the biological implications of this finding?

When considering the RNA as the substrate of molecular motors such as helicases and other remodeling enzymes, the results could likely be interpreted from an evolutionary point of view which could allow deciphering the basis of the evolutionary pressure responsible for the unwinding mechanism catalyzed by RNA-duplex processing enzymes.

The RNA unwinding catalyzed by helicases is coupled to adenosine triphosphate (ATP) binding and hydrolysis. The underlying mechanism would reasonably minimize the use of ATP, especially in a low-nutrient environment. Provided that the intrinsic RNA dynamics implies that at ss-ds junctions the unbinding of the $5^{\prime}$-strand base is favored over the unbinding of the complementary $3^{\prime}$-strand base, an enzymatic unwinding model would include a mechanism in which the separation of the two complementary strands is accom- plished by acting on the weakest portion, i.e. the 5 '-strand base. Thus, an ancestral enzyme using the $3^{\prime}$-strand as running track rail (with 3' to 5'directionality) without perturbing its conformation and causing the displacement of the $5^{\prime}$-strand by mechanical exclusion could satisfy some energysaving requirements.

The viral RNA helicase NS3 of hepatitis C virus, which is a prototypical $\mathrm{DEx}(\mathrm{H} / \mathrm{D})$ RNA helicases essential for viral replication, could satisfy the above mentioned requirements. ${ }^{445}$ NS3 is a potentially relevant drug target and has been structurally and functionally characterized in various contexts. ${ }^{48-51}$ It unwinds duplexes by first loading onto a single-stranded $3^{\prime}$-terminus region and then processively translocating with $3^{\prime}$ to $5^{\prime}$ directionality along this loading strand, thereby peeling off the complementary $55^{\prime}$-strand bases. In particular, the $3^{\prime}$-strand would migrate through a tracking ssRNA tunnel running within the protein whereas the complementary $5^{\prime}$-strand is forced towards the back of the protein by the "helix opener" hairpin (6). ${ }^{5253}$ In light of the free-energy calculations discussed above, it could be suggested that this mechanism has been optimized according to the intrinsic RNA unwinding dynamics disclosed in this work.

Arguably, the processing machineries are being constantly shaped by the evolutionary pressure of a plethora of (often unknown) factors contributing to the optimization of metabolism in the whole living system, rather than to the local biochemical process. As a consequence, the preference for a welldefined RNA processing directionality cannot be 
ubiquitously observed. .45

We speculate that all the biochemical processes involving RNA in which directionality plays a role (e.g. transcription), could be related to the energetics of RNA double helix forming and fraying discussed in this Article.

\section{Conclusions}

This study lays down the basis for the molecularlevel understanding of intrinsic RNA dynamics and its role in function. The asymmetric behavior of the $3^{\prime}$ - and $5^{\prime}$-strand could be responsible for the directionality observed in RNA processing. From a computational perspective, the approach we introduced can be generalized to analyze any kind of (un)binding event. Indeed, it allowed the free-energy landscape to be reconstructed along different reaction coordinates and the unbinding energies to be easily computed in an automatic and user-independent manner, therefore removing statistical and human biases. We foresee the application of our approach to a wider range of molecular systems, including the typical ligand-target complex faced in drug discovery. 54

\section{Acknowledgment}

We thank Francesco Di Palma, Rolando Hong and Vittorio Limongelli for critically reading the manuscript and an anonymous referee for several useful suggestions. We acknowledge the CINECA Award N. HP10BLIT9Z, 2011 for the availability of high performance computing resources and MIUR grant "FIRB - Futuro in Ricerca” N. RBFR102PY5 for funding. Developed software is available on request.

Supporting Information Available: Details of methodology and computations. Unbinding freeenergy profiles for both terminal and non-terminal base pairs. Further discussion on the comparison of computed and experimental dangling-end stabilities. This material is available free of charge via the Internet at http://pubs . acs . org/.

\section{References}

(1) Bloomfield, V. A.; Crothers, D. M.; Tinoco, I. J. Nucleic Acids: Structures, Properties, and Functions; University Science Books: Sausilito, CA, 2000.

(2) Haller, A.; Soulière, M. F.; Micura, R. Acc Chem Res 2011, 44, 1339-1348.

(3) Rocak, S.; Linder, P. Nat. Rev. Mol. Cell Biol. 2004, 5, 232-241.

(4) Jankowsky, E.; Fairman, M. Curr. Opin. Struct. Biol. 2007, 17, 316-324.

(5) Pyle, A. M. Annual Review of Biophysics 2008, 37, 317-336, PMID: 18573084.

(6) Norberg, J.; Nilsson, L. Acc. Chem. Res. 2002, 35, 465-472.

(7) Zhuang, Z.; Jaeger, L.; Shea, J. Nucleic Acids Res. 2007, 35, 6995.

(8) Li, P.; Vieregg, J.; Tinoco Jr, I. Annu. Rev. Biochem. 2008, 77, 77-100.

(9) Al-Hashimi, H.; Walter, N. Curr. Opin. Struct. Biol. 2008, 18, 321-329.

(10) Orozco, M.; Noy, A.; Pérez, A. Curr. Opin. Struct. Biol. 2008, 18, 185-193.

(11) Xia, T. Curr. Opin. Chem. Biol. 2008, 12, 604-611.

(12) Rinnenthal, J.; Buck, J.; Ferner, J.; Wacker, A.; Fürtig, B.; Schwalbe, H. Acc Chem Res 2011, 44, 1292-1301.

(13) Várnai, P.; Lavery, R. J. Am. Chem. Soc. 2002, 124, 7272-7273.

(14) Hagan, M. F.; Dinner, A. R.; Chandler, D.; Chakraborty, A. K. Proc. Natl. Acad. Sci. U. S. A. 2003, 100, 13922-13927.

(15) Giudice, E.; Lavery, R. J. Am. Chem. Soc. 2003, 125, 4998-4999.

(16) Mohan, S.; Hsiao, C.; VanDeusen, H.; Gallagher, R.; Krohn, E.; Kalahar, B.; Wartell, R. M.; Williams, L. D. J. Phys. Chem. B 2009, 113, 2614-2623. 
(17) Lee, M.-K.; Gal, M.; Frydman, L.; Varani, G. Proc. Natl. Acad. Sci. U. S. A. 2010, 107, 9192-9197.

(18) Zhao, L.; Xia, T. Methods 2009, 49, 128135.

(19) Karplus, M.; McCammon, J. A. Nat Struct Biol 2002, 9, 646-652.

(20) Sotomayor, M.; Schulten, K. Science 2007, 316, 1144-1148.

(21) Jossinet, F.; Ludwig, T. E.; Westhof, E. Bioinformatics 2010, 26, 2057-2059.

(22) Bussi, G.; Donadio, D.; Parrinello, M. J. Chem. Phys. 2007, 126, 014101.

(23) Parrinello, M.; Rahman, A. J. Appl. Phys. 1981, 52, 7182-7190.

(24) Wang, J.; Cieplak, P.; Kollman, P. A. J. Comput. Chem. 2000, 21, 1049-1074.

(25) Jorgensen, W. L.; Chandrasekhar, J.; Madura, J. F.; Impey, R. W.; Klein, M. L. J. Chem. Phys. 1983, 79, 926.

(26) Pérez, A.; Marchán, I.; Svozil, D.; Sponer, J.; Cheatham III, T.; Laughton, C.; Orozco, M. Biophys. J. 2007, 92, 3817-3829.

(27) Darden, T.; York, D.; Pedersen, L. J. Chem. Phys. 1993, 98, 10089-10092.

(28) Hess, B.; Kutzner, C.; Van Der Spoel, D.; Lindahl, E. J. Chem. Theory Comput. 2008, 4, 435-447.

(29) Bonomi, M.; Branduardi, D.; Bussi, G.; Camilloni, C.; Provasi, D.; Raiteri, P.; Donadio, D.; Marinelli, F.; Pietrucci, F.; Broglia, R.; Parrinello, M. Comput. Phys. Commun. 2009, 180, 1961-1972.

(30) Jarzynski, C. Phys. Rev. Lett. 1997, 78, 2690.

(31) Gore, J.; Ritort, F.; Bustamante, C. Proc. Natl. Acad. Sci. U. S. A. 2003, 100, 12564 12569.

(32) Bonomi, M.; Barducci, A.; Parrinello, M. J. Comput. Chem. 2009, 30, 1615-1621.
(33) Hummer, G.; Szabo, A. Proc. Natl. Acad. Sci. U. S. A. 2001, 98, 3658-3661.

(34) Gupta, A.; Vincent, A.; Neupane, K.; Yu, H.; Wang, F.; Woodside, M. Nature Physics 2011, 7, 631-634.

(35) Crooks, G. E. Phys. Rev. E 2000, 61, 23612366.

(36) Williams, S. R.; Evans, D. J. Phys. Rev. Lett. 2010, 105, 110601.

(37) Kumar, S.; Rosenberg, J. M.; Bouzida, D.; Swendsen, R. H.; Kollman, P. A. J. Comput. Chem. 1992, 13, 1011-1021.

(38) Souaille, M.; Roux, B. Comput. Phys. Commun. 2001, 135, 40-57.

(39) Cantor, R.; Schimmel, P. Biophysical Chemistry; W. H. Freeman: San Francisco, 1980.

(40) Hummer, G.; Szabo, A. Acc. Chem. Res. 2005, 38, 504-513.

(41) Hummer, G.; Szabo, A. Proc. Natl. Acad. Sci. U. S. A. 2010, 107, 21441-21446.

(42) Mohan, S.; Hsiao, C.; Bowman, J. C.; Wartell, R.; Williams, L. D. J. Am. Chem. Soc. 2010, 132, 12679-12689.

(43) Liu, J. D.; Zhao, L.; Xia, T. Biochemistry 2008, 47, 5962-5975.

(44) Sugimoto, N.; Kierzek, R.; Turner, D. H. Biochemistry 1987, 26, 4554-4558.

(45) Turner, D. H.; Sugimoto, N.; Freier, S. M. Annual Review of Biophysics and Biophysical Chemistry 1988, 17, 167-192.

(46) Isaksson, J.; Chattopadhyaya, J. Biochemistry 2005, 44, 5390-5401.

(47) Pohorille, A.; Ross, W.; Tinoco, I. International Journal of Supercomputer Applications and High Performance Computing 1990, 4, 81-96.

(48) Dumont, S.; Cheng, W.; Serebrov, V.; Beran, R. K.; Tinoco Jr, I.; Pyle, A. M.; Bustamante, C. Nature 2006, 439, 105. 
(49) Büttner, K.; Nehring, S.; Hopfner, K.-P. Nat. Struct. Mol. Biol. 2007, 14, 647-652.

(50) Gu, M.; Rice, C. Proc. Natl. Acad. Sci. U. S. A. 2010, 107, 521.

(51) Raney, K. D.; Sharma, S. D.; Moustafa, I. M.; Cameron, C. E. J. Biol. Chem. 2010, 285, 22725-22731.

(52) Luo, D.; Xu, T.; Watson, R. P.; SchererBecker, D.; Sampath, A.; Jahnke, W.; Yeong, S. S.; Wang, C. H.; Lim, S. P.; Strongin, A.; Vasudevan, S. G.; Lescar, J. EMBO J. 2008, 27, 3209-3219.

(53) Serebrov, V.; Beran, R. K. F.; Pyle, A. M. J. Biol. Chem. 2009, 284, 2512-2521.

(54) Jorgensen, W. L. Nature 2010, 466, 42-43. 